\title{
Field-heated, field-cooled, and zero-field-heated static permittivity of the deuteron glass $\mathrm{Rb}_{1-x}\left(\mathrm{ND}_{4}\right)_{x} \mathrm{D}_{2} \mathrm{AsO}_{4}$
}

\author{
N. J. Pinto, ${ }^{*}$ K. Ravindran, ${ }^{\dagger}$ and V. H. Schmidt \\ Department of Physics, Montana State University, Bozeman, Montana 59717 \\ (Received 16 December 1992; revised manuscript received 31 March 1993)
}

\begin{abstract}
Dielectric permittivity and remanent polarization measurements have been performed in deuterated rubidium ammonium dihydrogen arsenate with $x=0.28$. Upon cooling the crystal, there is only the "transition" to the deuteron glass state. Static permittivity measurements were carried out along the $a$ tetragonal axis at two different heating and cooling rates. Applying an external dc electric field along the $a$ axis creates a bias which forces the acid deuteron in the O-D $\cdots O$ bond to lie at one end as opposed to the other as the temperature is lowered. This gives rise to a static permittivity ( $\approx$ polarization and/or field) which freezes in below the glass "transition" temperature $T_{g}$. On switching the external electric field off, a remanent polarization is observed which vanishes above $T_{g}$ as the "frozen" deuterons acquire more and more energy to overcome the random potential barriers. A theoretical fit with one adjustable parameter is seen to be in good agreement with the experimental results.
\end{abstract}

\section{INTRODUCTION}

Since the initial discovery that the deuterated ${ }^{1}$ family of materials with the general chemical formula $A_{1-x}\left(\mathrm{ND}_{4}\right)_{x} \mathrm{D}_{2} B \mathrm{O}_{4}$, where $A=(\mathrm{Rb}$ or $\mathrm{K})$ and $B=(\mathrm{P}$ or As) exhibit a deuteron glass state for certain values of $x$, similar to the proton glass state observed in phosphates ${ }^{2}$ and arsenates, ${ }^{3}$ many experimental techniques have been applied in order to clearly delineate the nature of these and the most recent proton glass system, BP-BPI. ${ }^{4}$ When constituents of a pure ferroelectric and a pure antiferroelectric are mixed in a certain ratio range, the frustration between ferro- and antiferroelectric interactions in the resulting crystal lead to glassy behavior. We report here our studies on deuterated rubidium ammonium dihydrogen arsenate (DRADA) with an ammonium concentration $x=0.28$. In this sample, rubidium dihydrogen arsenate is the ferroelectric and ammonium dihydrogen arsenate the antiferroelectric constituent. Competing ferroelectric and antiferroelectric interactions, together with random bias electric fields present in such systems ${ }^{5}$ due to the random placement of the $\mathrm{ND}_{4}$ groups, lead to a random freeze-out of the acid deuteron in the O-D $\cdots$ O bonds as temperature is lowered below $T_{g}$. The position of the deuteron in its double-well potential affects the equilibrium position of the As atom within the $\mathrm{AsO}_{4}$ tetrahedron and hence affects the type of ordering in the unit cell viz. ferroelectric or antiferroelectric.

A unified theory that encompasses the essential behavior of spin glasses, ${ }^{6}$ orientational glasses, ${ }^{7}$ deuteron $^{1}$ and/or proton ${ }^{2}$ glasses, polymer glasses, ${ }^{8}$ and canonical $\left(\mathrm{SiO}_{2}\right)$ glasses is still an open problem. In this paper we report field-cooling, field-heating, and zero-fieldheating experiments in addition to dielectric measurements on DRADA with $x=0.28$. Our dielectric measurements supplement the data reported by Schmidt et al., ${ }^{9}$ while field-cooling experiments were performed analogous to those reported by Levstik et al. ${ }^{10}$ on the deuterated phosphate glass. Prior to this, Hessinger and Knorr ${ }^{11}$ carried out field-cooling experiments on quadrupolar glasses.

The purpose of these experiments is to establish a link between spin glasses and canonical glasses. Common to spin and canonical glasses is the absence of a random dc bias which is present in quadrupolar and deuteron glasses. The dielectric response of quadrupolar and deuteron glasses are affected by the presence of external electric fields. This response below the ergodic limit to an external electric field depends on the history of the system, i.e., how the low-temperature phase was reached. If the system is cooled in zero field and then the field is switched on, the corresponding field-heated permittivity $\epsilon_{\mathrm{FH}}(T)$ differs from the field-cooled permittivity $\epsilon_{\mathrm{FC}}(T)$ obtained by cooling the sample in the presence of an external field to temperatures below the glass "transition" temperature. Above $T_{g}$ the two would be the same.

\section{EXPERIMENT}

The crystal used in this experiment was grown by $Z$. Trybula by slow evaporation of an aqueous solution of $\mathrm{RbD}_{2} \mathrm{AsO}_{4}$ (DRDA) and $\mathrm{ND}_{4} \mathrm{D}_{2} \mathrm{AsO}_{4}$ (DADA) mixed in the proper molar ratio. Small platelets of $2.71 \times 1.7 \times 0.9$ $\mathrm{mm}^{3}$ and $3.1 \times 2.4 \times 0.9 \mathrm{~mm}^{3}$ perpendicular to the $c$ and $a$ tetragonal axes, respectively, were cut from a single crystal. After polishing the faces, silver electrodes were evaporated to ensure good surface contacts.

Dielectric measurements were carried out in a model RW3 Leybold-Heraeus cold head along the $a$ and $c$ axes. The sample was cooled and the complex dielectric constant $\epsilon^{\prime}-i \epsilon^{\prime \prime}$ was measured using a Model 625 WayneKerr component analyzer. Temperature was measured using a Model 330 Lakeshore temperature controller with a silicon diode sensor.

The field-cooling experiments were carried out with electric field along the $a$ axis. The crystal was placed in an Oxford Instruments model ESR-900 continuous-flow 
cryostat. A model 610R Keithley electrometer was connected across a reference capacitor $(15 \mathrm{nF})$ placed in series with the crystal. A small leakage of charge through the electrometer resulted in the curves deviating from the expected behavior for the static permittivity. Temperatures were measured using a calibrated Chromel-Alumel-type $\mathrm{K}$ thermocouple.

\section{RESULTS AND DISCUSSION}

Figures 1(a) and 1(b) show the real and imaginary part of the dielectric constant $\epsilon^{\prime}-i \epsilon^{\prime \prime}$ measured along the $a$ axis, while Figs. 2(a) and 2(b) show the same quantities measured along the $c$ axis. Both figures show the presence of a glass state at low temperatures with no ferroelectric or antiferroelectric transition. It is interesting to note that the values of $\epsilon^{\prime}$ and $\epsilon^{\prime \prime}$ are found to be greater along the $a$ axis than along the $c$ axis. A similar effect is $\operatorname{seen}^{3}$ in the undeuterated counterpart. The glass transition temperature at the lowest frequency of $100 \mathrm{~Hz}$ is $\approx 50 \mathrm{~K}$ as can be seen in Figs. 1(a) and 2(a). It is defined as the temperature at which $\epsilon_{a}^{\prime}$ begins to drop more rapidly with decreasing temperature for that frequency. The glass transition is more gradual along the $a$ axis than along the $c$ axis and is smeared due to the presence of random fields. ${ }^{5}$ Upon cooling into the glass state the acid deuteron freezes at random into one of the two O-D $\cdots O$ bond sites. The position of the acid deuteron within the bond is influenced by the presence of the neighboring $\mathrm{ND}_{4}$ groups, which are placed randomly in some of the surrounding cation sites. This tilts the double-well potential barrier resulting in a random effective local electric field. ${ }^{5}$

The dispersion in the dielectric constant below $T_{g}$ is a
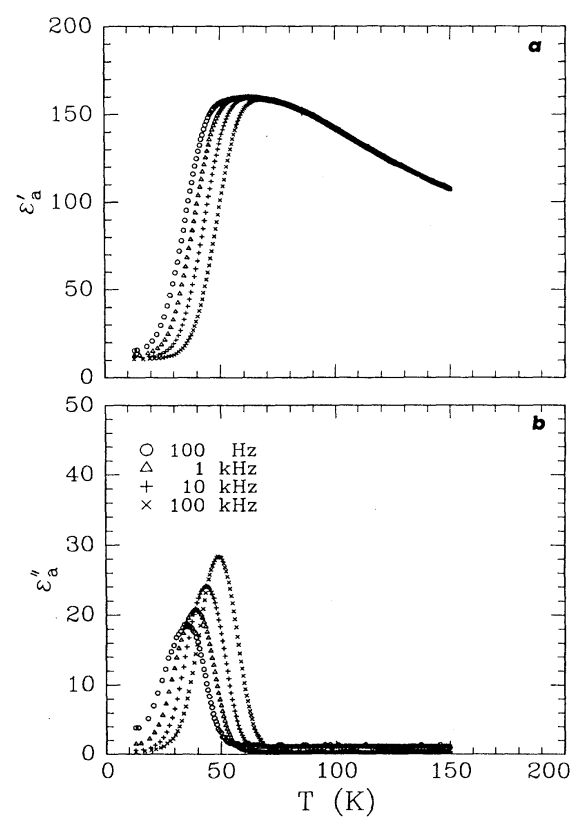

FIG. 1. Temperature dependence of the (a) real part $\epsilon_{a}^{\prime}$ and the (b) imaginary part $\epsilon_{a}^{\prime \prime}$ of the dielectric permittivity along the $a$ axis in the deuteron glass regime for DRADA with $x=0.28$.

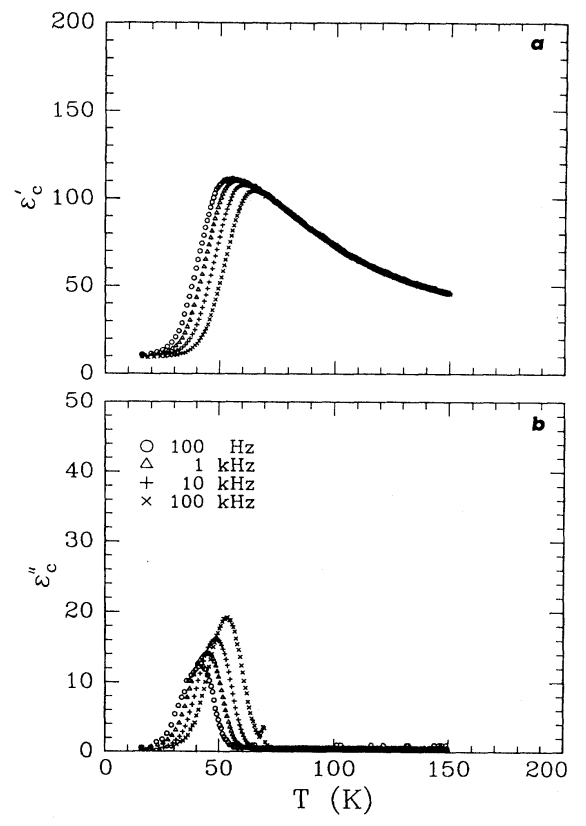

FIG. 2. Temperature dependence of the (a) real part $\epsilon_{c}^{\prime}$ and the (b) imaginary part $\epsilon_{c}^{\prime \prime}$ of the dielectric permittivity along the $c$ axis in the deuteron glass regime for DRADA with $x=0.28$.

feature characteristic of proton and/or deuteron glasses. There exists in this region a spread of relaxation times which result from the fractal nature of the effective potential in which $\mathrm{HAsO}_{4}$ and $\mathrm{H}_{3} \mathrm{AsO}_{4}$ "bound chargecarrier" groups in effect diffuse by means of intrabond deuteron transfer. ${ }^{12,13}$ This diffusion is responsible for the dielectric relaxation.

The effective diffusion of these groups in this fractal effective potential offers a simple spatial explanation for the nonergodic behavior evidenced by field-cooling, fieldheating, and zero-field-heating experiments in proton glass crystals. Below the ergodic temperature $T_{e}$ the random potential barriers encountered by these groups are so high that they cannot find partners with which to recombine on any reasonable time scale. As temperature drops, the region accessible to their diffusion on a reasonable time scale becomes smaller and smaller, and any polarization in the unvisited portion of the crystal is frozen in, independent of subsequent change in applied field $E$. The maximum barrier encountered is proportional to the square root of the distance diffused because the individual potential steps are randomly up or down. At a given temperature $T$, the diffusion distance possible in a reasonable time is thus proportional to $T^{2}$. The volume available to the diffusing group is then proportional to $T^{6}$.

To apply this idea to our experimental results, we consider first the field-cooled (FC) runs shown in Figs. 3 and 4. A sizable polarization is frozen in and remains unchanged as $T$ falls below $T_{e}$. Then at low temperature the dc field $E$ is removed, and the polarization immediately drops by an amount $-\epsilon_{0} \epsilon_{\infty} E$, where $\epsilon_{0}$ is the mks 


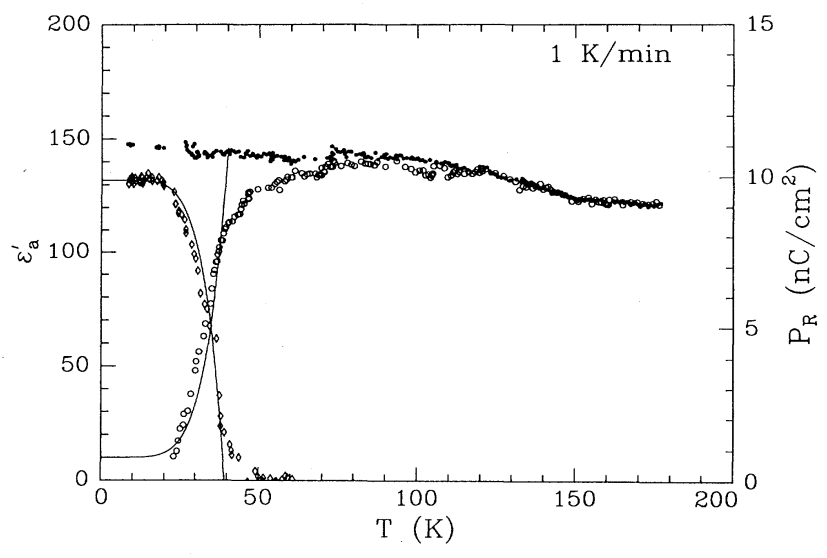

FIG. 3. Temperature dependence of the field-heated $(O)$ and field-cooled (๑) static delectric constant (left scale) of DRADA with $x=0.28$ measured along the $a$ axis. The remanent polarizatrion $P_{R}(\diamond$, zero-field heating) is also shown (right scale). The electric field applied was $500 \mathrm{~V} / \mathrm{cm}$ and the heating and cooling rate was $1 \mathrm{~K} / \mathrm{min}$. Solid lines represent fit to the theory.

constant and $\epsilon_{\infty}$ is that part of the relative permittivity due to the vacuum, electronic, and fast ionic response which is independent of deuteron intrabond motion. As the crystal is then warmed up in zero field $(\mathrm{ZFH}=$ zerofield-heating run), the carriers are at first almost completely trapped and the polarization does not drop appreciably. With further heating, the amount of drop becomes noticeable and obeys the $P=P_{i}\left[1-\left(T / T_{e}\right)^{6}\right]$ law quite accurately. As $P$ drops toward zero, the rate of decay with temperature decreases. This tail, not predicted by the $T^{6}$ law, is due to the random spacing of the trapped carriers. This leaves some polarized islands unvisited by carriers even above $T_{e}$ where the carriers begin to meet their annihilation partners and the material overall exhibits ergodic behavior again.

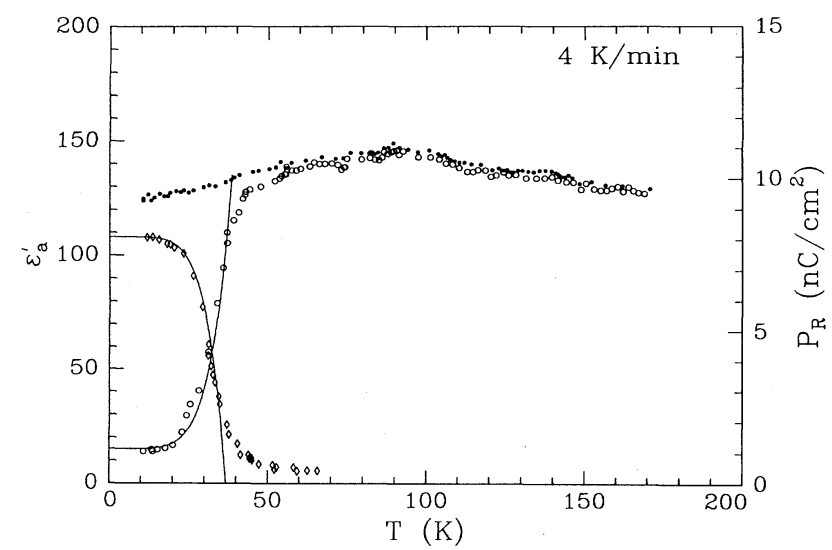

FIG. 4. Temperature dependence of the field-heated $(O)$ and field-cooled $(\bullet)$ static delectric constant (left scale) of DRADA with $x=0.28$ measured along the $a$ axis. The remanent polarizatrion $P_{R}(\diamond$, zero-field heating) is also shown (right scale). The electric field applied was $500 \mathrm{~V} / \mathrm{cm}$ and the heating and cooling rate was $4 \mathrm{~K} / \mathrm{min}$. Solid lines represent fit to the theory.
Similar analysis applies to the field-heating (FH) runs, which actually occurred earlier in our experimental sequence. We first cooled the crystal in zero field. Then at the lowest temperature we turned on the dc field $E$ and observed the immediate "infinite-frequency" polarization jump of magnitude $\epsilon_{0} \epsilon_{\infty} E$. Upon subsequent heating in the FH runs, the polarization at first hardly changes but then starts rising, obeying a $P=P_{f}-\left(P_{f}-P_{i}\right)$ $\times\left[1-\left(T / T_{e}\right)^{6}\right]$ law, where $P_{i}$ is the initial polarization from the infinite-frequency response and $P_{f}$ is the final polarization when the crystal becomes ergodic.

The results of these fits are $T_{e}=39.0 \mathrm{~K}$ for the 1 $\mathrm{K} / \mathrm{min} \mathrm{ZFH}$ run, $T_{e}=39.9 \mathrm{~K}$ for the $1 \mathrm{~K} / \mathrm{min} \mathrm{FH}$ run, $T_{e}=36.9 \mathrm{~K}$ for the $4 \mathrm{~K} / \mathrm{min} \mathrm{ZFH}$ run, and $T_{e}=38.5 \mathrm{~K}$ for the $4 \mathrm{~K} / \mathrm{min} \mathrm{FH}$ run. We expected $T_{e}$ to be lower by about $1.5 \mathrm{~K}$ for the slower temperature sweep rate, so we can only say that $T_{e}$ defined by the above relations is about $38 \pm 2 \mathrm{~K}$ for sweep rate in the $1-4 \mathrm{~K} / \mathrm{min}$ range.

Predicted values of $T_{e}$ for this sweep rate based on our model $^{12,13}$ for carrier diffusion in a fractal potential are in the $30-50 \mathrm{~K}$ range, depending on the values chosen for carrier creation energy and size of random step in potential energy along the diffusion path. Details of this calculation are beyond the scope of this paper and will be presented elsewhere.

We now provide some additional discussion of Figs. 3 and 4, which show the temperature dependence of the field-heated, field-cooled, and zero-field-heated static permittivity of a DRADA $x=0.28$ crystal along the $a$ axis for two different heating and cooling rates. The crystal was first cooled in zero field to about $8 \mathrm{~K}$, then a dc field of $500 \mathrm{~V} / \mathrm{cm}$ was switched on. The corresponding fieldheated susceptibility $\chi_{\mathrm{FH}}(T)=\lim _{E \rightarrow 0} P(E, T) / \epsilon_{0} E$ is then computed knowing the charge stored on the crystal. $P$ is the polarization and $E$ is the electric field. The sample was then heated through the glass transition and then cooled at the same rate with the field left on. The corresponding field-cooled susceptibility $\chi_{\mathrm{FC}}(T)$ $=\lim _{E \rightarrow 0} P(E, T) / \epsilon_{0} E$ is calculated likewise. From these results the corresponding dielectric permittivities $\epsilon_{\mathrm{FH}}$ and $\epsilon_{\mathrm{FC}}$ were computed. The field was then switched off and a remanent polarization is observed which decays as a function of temperature. The corresponding zero-fieldheated susceptibility was calculated. Static permittivity measurements corresponding to these various stages are seen in Figs. 3 and 4.

We distinguish, in Figs. 3 and 4, three regions of interest with different responses to the application of an electric field. The paraelectric phase above $60 \mathrm{~K}$ is monodispersive as the deuterons are mobile enough to follow the exciting electric field even at high frequencies, as is evident from Figs. 1(a) and 2(a). Below $20 \mathrm{~K}$ the system is completely nonergodic as the deuterons are completely frozen in their bond sites. From 20 to about $60 \mathrm{~K}$, thermal motion allows at least some of the acid deuterons to move within their bonds. The system becomes ergodic in this region as temperature rises, and exhibits dielectric relaxation with frequency dispersion.

Initially, when the system is cooled without an external field the random freezing of the acid deuterons results in zero polarization. Upon applying an external electric 
field at low temperature, the infinite-frequency response occurs immediately. As temperature increases, the deuteron system begins to respond and the polarization builds up and reaches the field-cooled value when the system becomes ergodic, so that $\epsilon_{\mathrm{FC}}(T)=\epsilon_{\mathrm{FH}}(T)$. There is some deviation from the Curie-Weiss curve even above the ergodic temperature because of random bias tending to freeze out the response. This freezing causes $\epsilon_{\mathrm{FC}}(T)$ to tend to become horizontal below $T_{g}$ as $T \rightarrow 0$, over and above the effects of nonergodicity. In the temperature range $T<T_{g}, \epsilon_{\mathrm{FC}}(T)>\epsilon_{\mathrm{FH}}(T)$. The field-heated permittivity in this case is similar to the low-frequency limit of the ac permittivity which exhibits a rounded maximum as seen in Figs. 1(a) and 2(a). The field-cooled permittivity retains the same value with a slight change of slope as temperature is lowered below $T_{g}$, and continues to remain constant as temperature is decreased, due to the gradual freeze-out of the acid deuteron in the O-D $\cdots$ O bond. A remanent polarization is observed upon switching the field off. This polarization vanishes as temperature is raised above $T_{g}$. These results are very similar to those for spin glasses and support the evidence that the deuteron glass is spin-glass-like.

The cooling and subsequent heating runs for both temperature sweep rates suffer from electrometer drift. In Fig. 3 the drift in permittivity is upward, while in Fig. 4 it is downward. If one compensates for these drifts by simply superimposing corresponding curves for the two rates, there is no noticeable polarization growth or decay lag at the higher sweep rate.

Comparison of the $\mathrm{FC} / \mathrm{FH}$ results with the ac permittivity results at temperatures for which these results should agree, namely above the onset of dispersion in the ac measurements and above the $\mathrm{FC} / \mathrm{FH}$ permittivity separation point, shows small discrepancies. The FC/FH $a$ axis permittivity peak of 145 (Figs. 3 and 4) is between 75 and $90 \mathrm{~K}$, while the $a$-axis ac peak of 160 [Fig. 1(a)] is between 60 and $70 \mathrm{~K}$. The difference in permittivity peak values is not unusual, and can be attributed to error in measuring contact area and crystal thickness, and perhaps to electrode quality. Poor thermal contact between the sample and the cold head resulted in lower than actual temperatures reported for the dielectric permittivity. This was confirmed by mounting a KDA $\left(\mathrm{KH}_{2} \mathrm{AsO}_{4}\right)$ sample in the cold head and finding $T_{c}=87$ $\mathrm{K}$ instead of the actual value of $97 \mathrm{~K}$. Going up to $170 \mathrm{~K}$ near the maximum temperature for the FC/FH results, the dc permittivity has dropped by the same fraction that the ac permittivity drops in going up to $105 \mathrm{~K}$. This slower drop for dc permittivity is consistent with similar experiments on DRADP. ${ }^{1,10,14}$ This effect may originate in electrical or electrochemical interactions at the electrodes, or in long-lived ferroelectric clusters associated with impurities or defects. More experiments are being carried out on other mixed crystals to verify and find the origin of this effect.

\section{CONCLUSIONS}

Our results are qualitatively similar to those of Levstik et al. ${ }^{10}$ on DRADP. They used a temperature sweep rate of $1 \mathrm{~K} / \mathrm{min}$ and a field of $500 \mathrm{~V} / \mathrm{cm}$, both being values that we also used. They did not obtain a fieldheating (FH) curve, while we did not obtain a zero-field cooling (ZFC) curve. However, their ZFC technique should give exactly the same results as our FH method. Their ZFC technique seems unnecessarily time consuming, which may explain why they have no points below the temperature of $48 \mathrm{~K}$ where the polarization "tail" begins. They remark on an unexplained difference between $T_{f}=61 \mathrm{~K}$ where the $\mathrm{ZFC}$ tail ends and $52 \mathrm{~K}$ where the ZFH tail ends and the polarization reaches zero. This difference originates in the anomalously small tail for the ZFH run, compared to the four tails in our runs which are comparable in size to their ZFC tail. One would expect all these tails to have about the same size.

Fits of their ZFH and ZFC runs to our $T^{6}$-law expressions above give $T_{e}=47.0 \mathrm{~K}$ and $50.0 \mathrm{~K}$, respectively. Their data has less scatter than ours and appears not to suffer from electrometer drift problems. The fit to the $\mathrm{ZFC}$ run assumed that the $48-\mathrm{K}$ points represent the start of the tail, so it is only a fit to two points and gives no information on correctness of the $T^{6}$ law. The fit to their ZFH curve as seen in Fig. 5 is quite good, similar to our fit shown in Figs. 3 and 4 in that the measured points fall slightly below the best-fit curve in the middle of the temperature range. Accordingly, a power slightly lower than 6 would give a better fit.

The $T^{6}$ law fits their data and ours much better than the sloping line in their Fig. 1 representing the Sherrington-Kirkpatrick model ZFC prediction. Here we assume that their ZFC data would be a mirror reflection of their $\mathbf{Z F H}$ data across a horizontal line at $\epsilon^{\prime}=51.5$. The relation of the $T^{6}$ law, which so far is being applied only to proton glass, to the SherringtonKirkpatrick model, which has wide applicability but is based on certain approximations, is an interesting problem deserving study.

Further study is recommended, for proton glasses of other types and compositions, including $\epsilon_{c}$ as well as $\epsilon_{a}$

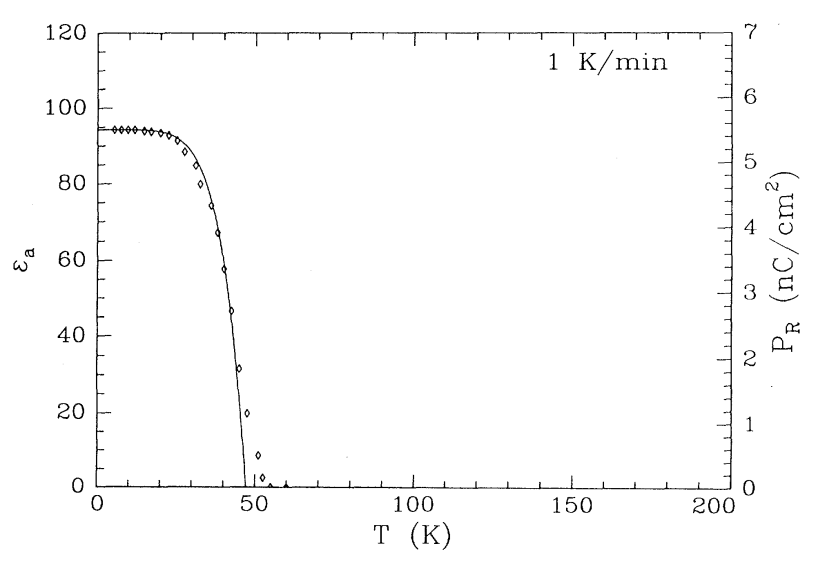

FIG. 5. Temperature dependence of the remanent polarization in $\mathrm{Rb}_{1-x}\left(\mathrm{ND}_{4}\right)_{x} \mathrm{D}_{2} \mathrm{PO}_{4}$ (DRADP) with $x=0.60$, taken from Ref. 10. The solid line represents a fit to the theory. 
measurements. Also, the reasoning behind the $T^{6}$ law predicts some specific behavior that could be checked. For instance, if the zero-field warming process were interrupted, perhaps near $33 \mathrm{~K}$ where the polarization has dropped to half its initial value, and the field were restored to its original value $E$, the polarization should rise quickly to its original value at low temperature before $E$ was removed. Conversely, if the field were changed from zero to $-E$, the polarization should drop to zero.

\section{ACKNOWLEDGMENTS}

The authors thank Professor J. E. Drumheller for the use of the low-temperature helium flow-through cryostat used in performing the field-cooled experiments, Dr. S. L. Hutton for his assistance and for automating the electronic apparatus used in performing this experiment, and Professor G. F. Tuthill for helpful discussions. This work was supported by National Science Foundation Grant No. DMR-9017429.
*Present address: Physics Department, Wichita State University, Wichita, KS 67208.

${ }^{\dagger}$ Present address: Physics Department, University of Cincinnati, Cincinnati, OH 45221.

${ }^{1}$ V. H. Schmidt, S. Waplak, S. Hutton, and P. Schnackenberg, Phys. Rev. B 30, 2795 (1984).

${ }^{2}$ E. Courtens, J. Phys. Lett. (Paris) 43, L199 (1982).

${ }^{3}$ Z. Trybula, V. H. Schmidt, J. E. Drumheller, D. He, and Z. Li, Phys. Rev. B 40, 5289 (1989).

${ }^{4}$ S. L. Hutton, I. Fehst, R. Bohmer, M. Braune, B. Mertz, P. Lunkenheimer, and A. Loidl, Phys. Rev. Lett. 66, 1990 (1991).

${ }^{5}$ R. Pirc, B. Tadić, and R. Blinc, Z. Phys. B 61, 69 (1985); R. Pirc, B. Tadić, and R. Blinc, Phys. Rev. B 36, 8607 (1987).

${ }^{6}$ K. Binder and A. P. Young, Rev. Mod. Phys. 58, 801 (1986).
${ }^{7}$ U. T. Höchli, K. Knorr, and A. Loidl, Adv. Phys. 39, 405 (1990).

${ }^{8}$ J. K. Krüger, R. Roberts, H.-G. Unruh, K.-P. Fruhauf, J. Helwig, and H. E. Müser, Prog. Colloid. Polym. Sci. 71, 77 (1985).

${ }^{9}$ V. H. Schmidt, Z. Trybula, D. He, J. E. Drumheller, C. Stigers, Z. Li, and F. L. Howell, Ferroelectrics 106, 119 (1990).

${ }^{10}$ A. Levstik, C. Filipič, Z. Kutnjak, I. Levstik, R. Pirc, B. Tadić, and R. Blinc, Phys. Rev. Lett. 66, 2368 (1991).

${ }^{11}$ J. Hessinger and K. Knorr, Phys. Rev. Lett. 65, 2674 (1990).

${ }^{12}$ V. H. Schmidt, J. Mol. Struct. 177, 257 (1988).

${ }^{13}$ V. H. Schmidt, Ferroelectrics 78, 207 (1988).

${ }^{14}$ M. Takashige, H. Terauchi, Y. Miura, and S. Hoshino, J. Phys. Soc. Jpn. 54, 3250 (1985). 